\title{
Modularity for Maximum Mobility and Manipulation: Control of a Reconfigurable Legged Robot with Series-Elastic Actuators
}

\author{
Simon Kalouche, David Rollinson, and Howie Choset
}

\begin{abstract}
We present a set of modular series-elastic actuators (SEAs) that allow rapid and robust prototyping of mobile legged robots. The SEA modules were originally developed for a snake robot, $S E A$ Snake, and have recently been reconfigured into Snake Monster, a multi-modal walking robot that can be easily adapted to hexapod, quadruped, and biped configurations. The use of SEAs allows the implementation of a compliant hybrid controller using both position and force-based walking. This paper presents the mechanical design, control architecture, and initial locomotion experiments using the Snake Monster platform. Additionally, we discuss the enhanced capabilities, pertaining particularly to search and rescue applications, enabled by the use of our modular hardware. Finally, we highlight how these modules provide a powerful tool for both field deployment requiring locomotion and manipulation tasks.
\end{abstract}

\section{INTRODUCTION}

First responder teams in urban search and rescue or disaster scenarios could benefit from a rapidly deployable robot with mission-specific configurations embodied within a compact, easily-transportable, package [1]. Disaster environments often contain unstructured, diverse, and challenging terrains and differ by case, exploiting the advantages of modular, reconfigurable robots that can be built in-situ to meet requirements of the specific task at hand. The goal of these robots is to provide first responders with the ability to remotely access and survey the disaster zone, locate victims, and possibly manipulate the environment [3][4].

Reconfigurable robots can bypass the complexity of specialized behaviors exhibited in animals competent of locomotion over varying terrain by allowing the robot operator to intelligently choose a task-specific configuration. In this way an entire set of robots with varying configurations, morphologies, and capabilities can be created using a modular approach. We believe this modular approach has many advantages over highly specialized robots that are harder to adapt to a wide range of different environments and tasks.

For instance a hexapod configuration can be used for collapsed, rubble-filled, environments where stability and navigability of terrain is prioritized. Alternatively, a multimodal quadruped with the ability to walk, or roll on wheels, offers both high mobility on rugged terrains in walking mode, as well as a higher efficiency for flat surfaces in rolling mode. Multi-modal platforms are advantageous over long distances over varying terrain. In man-made environments designed for humans, the larger form factor of hexapods and quadrupeds

*This work was supported by DARPA M3 program and the NDSEG Fellowship

${ }^{1}$ S. Kalouche, D. Rollinson and H. Choset are with Carnegie Mellon's Robotics Institute, School of Computer Science, Pittsburgh, PA 15206, USA chosetecs. cmu.edu

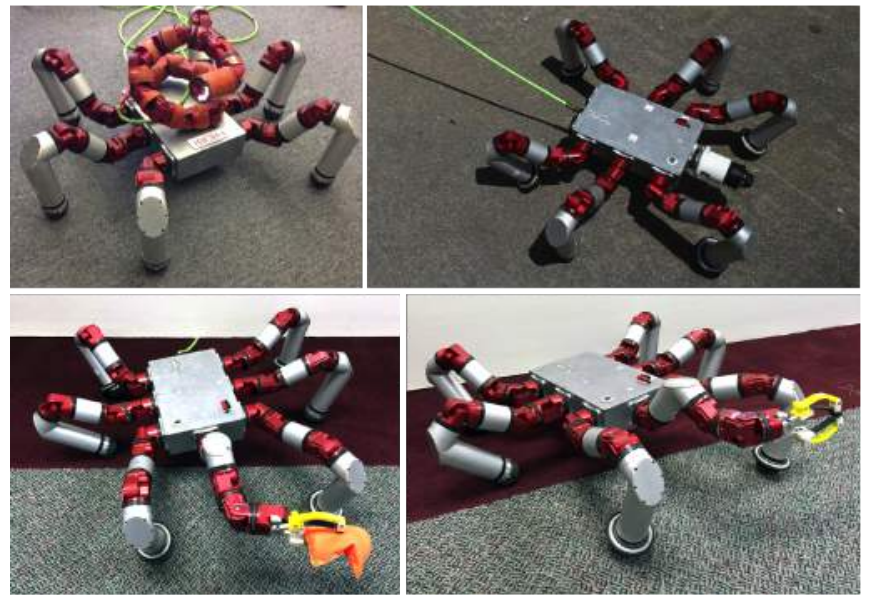

Fig. 1. Snake Monster (SM) with SEA Snake riding on its back (topleft). SM at the DRC Finals Expo using an IP camera head for live video streaming, object tracking, mapping, and navigation (top-right). SM configured with a 3-DoF arm and compliant, force-sensing, gripper (bottom)

may inhibit their mobility (stairs, narrow corridors, etc.). In these cases a biped configuration may be used. For very confined spaces or areas that have extremely limited access a snake robot could be the most useful configuration.

Although modular robots and legged robots both currently exist, there are limitations for each that prevent these technologies from becoming practical disaster response robots. Existing robots that offer forms of modularity are currently at relatively low technology readiness levels [6] and are meant to operate in regulated laboratory environments. These modular systems are often not fully sealed from the environment and do not contain robust, tool-less, electro-mechanical interfaces, rendering them difficult for rapid reconfiguration on the fly [7][8].

Similarly, limitations in limbed locomotion arise in robots that rely heavily on an accurate perception of the environment and precise interaction with that environment. As seen in the DARPA Robotics Challenge (DRC) Finals, when the current state-of-art robots were forced to operate in mock disaster environments in the absence of safety harnesses, these robots failed in many ways. The DRC exposed several weaknesses in current disaster response robots - in particular their lack of robustness in failure recovery [9]. Many robots fell during the competition, and these falls often resulted in serious damage. During the competition only one robot was able to fully recover without physical intervention [10]. To mitigate the challenges associated with failure recovery an alternative approach is to minimize the inherent possibility 
of failures. We propose that through the use of reliable hardware, a limited dependency on high-fidelity environmental perception, and control algorithms that are robust to unexpected perturbations, robots will be more resilient and thus useful in unpredictable, real-world scenarios [12].

In this respect, we present the Snake Monster robot, Fig. 1, as a reconfigurable search and rescue robot built with reliable field-tested hardware [11]. By using parallel position and force control, Snake Monster is able to actively and passively conform its footsteps to traverse unmodeled terrain with relative ease and minimal computation. Using the mechanical spring compliance built into each joint, Snake Monster conforms to uneven terrain [22] rather than explicitly planning and optimizing footholds and step locations. In this sense Snake Monster exhibits locomotive traits that are compliant like the extremely versatile RHex robot [13] as well as dexterous like the LittleDog robot [15][16].

\section{MOdUlar Design}

\section{A. Mechanical}

The SEA Snake and Snake Monster robots consist of actuated, 1-DoF modules shown in Fig. 2. Each module is a self-contained joint allowing for $180^{\circ}$ of rotation. The modules have distinct proximal and distal electromechanical, intermodule interfaces which are used to fasten two adjacent modules. Modules are mechanically connected by a simple threaded collar which is hand tightened to the female threads on the adjacent module. Dowel pins and matching recesses ensure the modules are mated and clocked correctly. Electrical connections between modules are made using spring-pin connectors and conductive target areas. This design enables modules to be connected and disconnected quickly and repeatedly [19][20].

The module housing is machined from 7075 aluminum and anodized to prevent wear and corrosion. O-rings laid in machined grooves seal the module at each interface meeting IP66 standards (splash-proof). The connections are secure and resist shock and stress [19]. The modules are driven by a modified Maxon EC 20 flat motor with a 349:1 gear reduction offering increased torque density within the package. The modules produce a maximum of $7 \mathrm{~N}-\mathrm{m}$ of torque and a maximum speed of $33 \mathrm{rpm}$ [19].

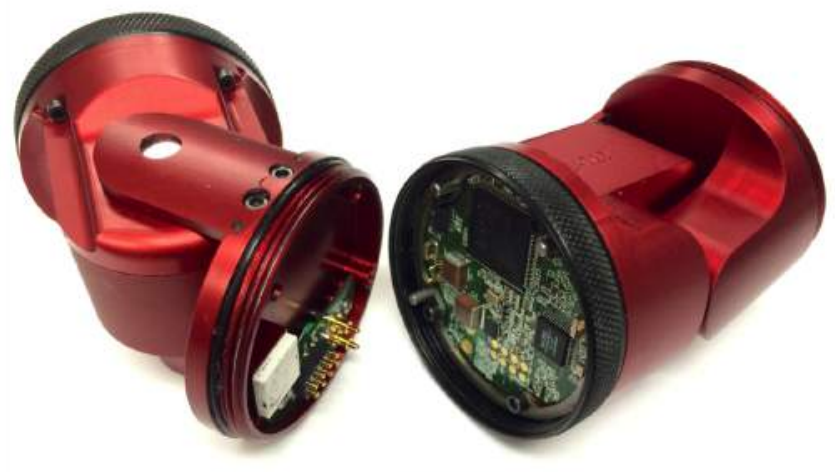

Fig. 2. Modular Electro-mechanical interfaces.

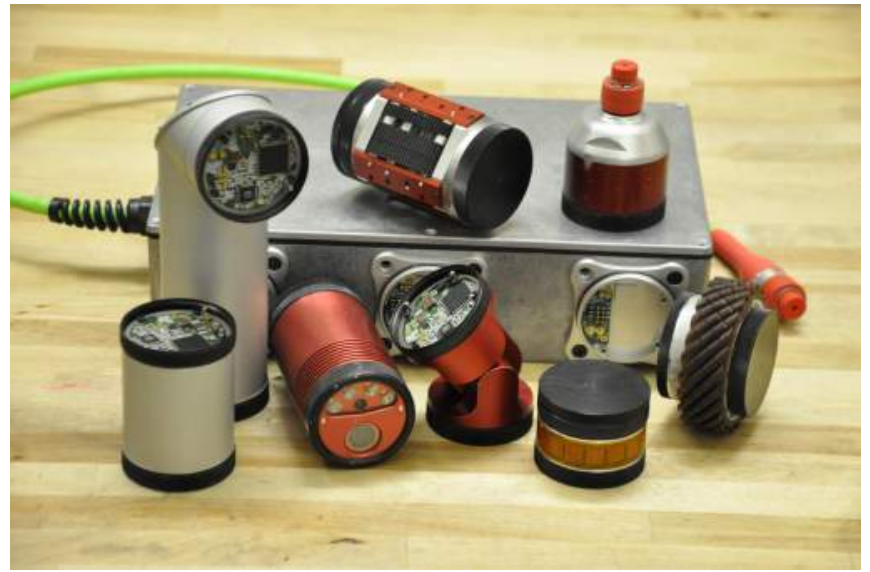

Fig. 3. Original SEA module and several other specialized actuation and sensing modules including tread, mecanum wheel, and 2-D Laser Distance Sensor Head modules among others.

Additionally, the 1-DoF rotary modules feature a serieselastic element which offers inherent mechanical compliance as well as torque sensing capabilities [22]. The 60A durometer natural rubber torsional spring with tapered cross-section has an approximate spring constant of $12 \mathrm{~N}-\mathrm{m} / \mathrm{rad}$ and a maximum rotational deflection of approximately 0.6 radians [20]. Because the stiffness of the rubber gradually changes as it is mechanically and thermally stressed, the spring constant is continually re-calibrated online using a recursive estimator and heuristic model based on motor current [18].

Any specialized module design consisting of sensors, actuators, and mechanisms is compatible with all other modules so long as the design adheres to the same simple electromechanical interface and software protocol. Examples of several specialized modules can be see in Fig. 3 and 6.

\section{B. Electronics}

Each module contains embedded electronics that include a 32-bit ARM Cortex processor and transmits data over 100Mbps Ethernet network using standard UDP/TCP. Adjacent modules also communicate with each other over serial connections for configuration discovery. Additionally, the control board also contains a motor driver chip, temperature, current, and voltage sensors used for homeostasis. An LED projects light through a transparent bolt in the module housing to provide immediate feedback on module status [19]. An internal sensor board contains two magnetic encoders as well as a 9-axis inertial measurement unit (IMU) for sensing output rotor angle, torque via spring deflection, and gravitational orientation. A distal interface board is used only as a means of transferring power and data over to the control board of the next module in the robot via gold-plated springpins and matching conductive target areas.

\section{Low-Level Motor Control}

The brushless DC motor in each joint is controlled using a cascaded PID controller with position, velocity, and torque inputs. Three feedback loops around each of these input combine to control the PWM signal sent to the motor. This 


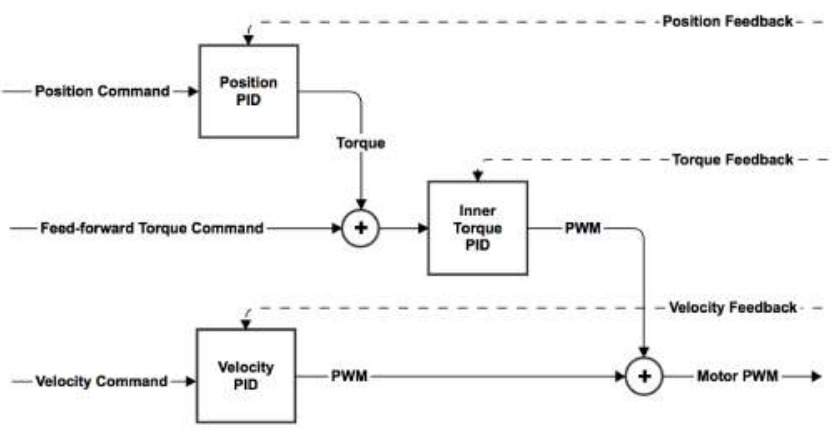

Fig. 4. Low-level cascade PID control strategy schematic block diagram.

control strategy enables the weight of each input to be finely tuned to favor one control input over another for precise position, velocity, or force control or for a balanced combination of all three. A schematic diagram of the cascade controller is shown in Fig. [4]. Note that the velocity controller feeds directly into motor PWM, rather than the torque controller. This is advantageous in that noisy velocity feedback signals do not get commanded to the torque controller allowing a strong feed-forward PWM to be calculated directly based on desired motor velocity.

Individual PID gains for each of the inputs can be tuned online from the high-level programming environment, which includes support for Java, Matlab, C++, and ROS.

Additionally, a low-level, closed-loop, 2-resistor thermal model is used to estimate winding and motor core temperatures to saturate the PWM signal and prevent motor burnout [21]. Using this thermal model, the module's nominal $5 \mathrm{~W}$ motors can be safely over driven to a peak power of over 30W. This allows us achieve short bursts of high power output from the motor and gradually limit the maximum PWM to keep the motor windings within their thermal limits.

\section{CONFIGURATIONS}

The intelligent, building-block, modules can be rapidly configured to suit a desired task. In this study Snake Monster was configured into an 18-DoF Hexapod, a 16DoF quadruped roller-walker, and a 14-DoF Biped. Initial locomotion and balancing experiments were conducted on each legged platform.

The hexapod is configured with three legs on opposite sides of a rectangular aluminum body box which houses an Ethernet network switch. Each port in the switch is connected to the chassis interface which transmits data to and from the leg modules. Each leg has three degrees of freedom allowing for position but not orientation control of the foot. A specialized sensing foot module was designed using the same electro-mechanical interface and is simply connected to the distal end of the limb as if it were another SEA module. This sensing module houses a single-axis force sensitive resistor (FSR) to measure ground reaction forces at each leg.

The quadruped is configured to have four legs -2 on either side of the same body box. Each of the four legs are augmented with an additional degree of freedom at the ankle for orientation of the foot as seen in Fig. 5. Inspiration for this configuration was derived from Hirose's Roller-Walker robot which also has 4-DoF limbs and can orient the ankle joint to toggle between multiple modes of mobility: walking and rolling [17]. Each of the 4 foot modules was modified with an external thin-section ball bearing whose inner race is press fit to the outer diameter of the foot module. A rubber o-ring tire is installed around a machined aluminum rim. The bearing introduces a passive torsional degree of freedom at the end-effector. With this modified foot module and ankle joint, Snake Monster can change the effective ground contact element between its static rubber foot or its passive wheel.

In addition to the hexapod and quadruped configurations, a biped is also constructed (Fig. 8). The biped is kinematically configured to have 5-DoF legs and 2-DoF arms. The 2DoF arms allow the biped to shift its center of mass $(\mathrm{CoM})$ and induce inertial forces for balancing laterally and longitudinally. The legs are identical in configuration and each consist of 2 hip joints, 1 knee joint, and 2 ankle joints. The hip and ankle joints provide adduction/abduction and flexion/extension motions while the knee only provides flexion/extension. A passive $90^{\circ}$ leg segment is configured in series between the hip joint and the knee joint to improve the workspace of the limb for walking and balancing. The biped is fit with specialized foot modules containing a $2 \times 2$ array of custom single-axis force sensitive resistors. The foot has a 0.25 " durable rubber sole used to protect the sensors and conform to small perturbations in the ground. The foot sensor, shown in Fig. 6 was designed to provide ground reaction force and center of pressure $(\mathrm{CoP})$ measurements.

\section{High-LeVel Control}

The high-level control for each of the legged configurations is based off of a simultaneous position and force control strategy. The main control loop operates at $50-100 \mathrm{~Hz}$ while the low-level motor control loop operates at $1 \mathrm{kHz}$. With every pass through the high-level loop the controller runs a simple state estimator to update body pose and track center of mass. The balancing controller calculates and outputs a position, velocity, and torque command for each joint on the robot. The position, velocity, and torque vectors (size $n \times 1$
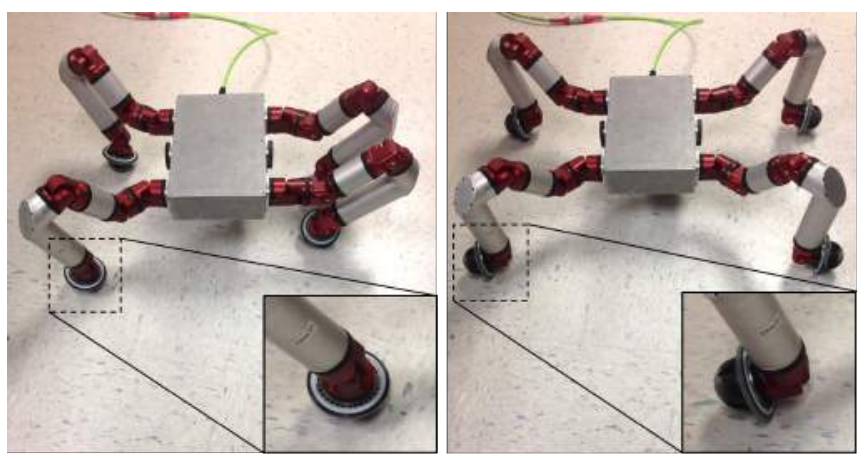

Fig. 5. Snake Monster is reconfigured into a quadruped with 4-DOF limbs, using the ankle joints to orient the end-effector to be a foot (left) or a passive skating wheel (right). 


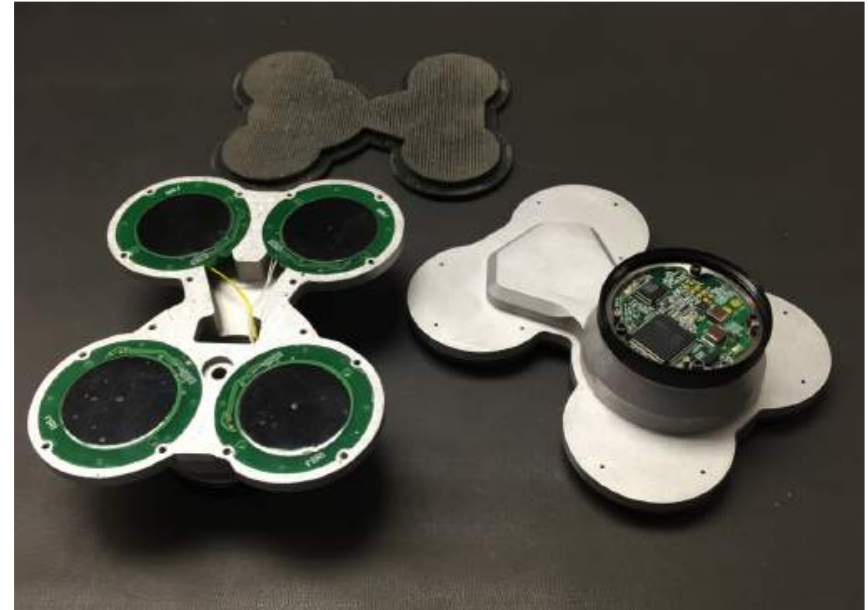

Fig. 6. Design of a lightweight (300 gram) multiple axis force sensing biped foot module.

where $n$ is the actuated degrees of freedom) are then sent to the low-level controllers running on the internal ARM processors of each SEA module.

The robots are semi-autonomous and can be tele-operated using a standard gaming controller. The operator control was deliberately designed to be easy and intuitive. The operator drives the robot as if it were an RC car using the joystick only to specify angular and linear velocities of the chassis, leaving the autonomous high and low level controllers with the details of balancing and walking.

\section{HEXAPOD}

\section{A. Gain Scheme}

The hexapod's high-level controller first initializes and sets the low-level cascade PID gains for position, velocity, and torque loops. To achieve a compliant stance sensitive to the environment and external disturbances, the position gains are set relatively low in comparison to the torque gains. This gain scheme achieves compliance by emphasizing joint torques that support the weight of the body in stance rather than joint positions which merely command the foot to a specific position. This loose position servoing enables the body to be pushed or pulled in any direction relative to stationary stance feet.

If the position gains are set high, the low-level controller prioritizes position over other control inputs and thus follows foot trajectories much tighter. If the robot were pushed and pulled using this high-position gain scheme, the entire robot would consequently drag along the surface of the ground while the controller works to hold a fixed position of the feet relative to the body. Therefore, comparative experiments demonstrate that relying heavily on position commands significantly decreases the robots ability to adapt to disturbances and environmental forces.

\section{B. State Estimation}

The hexapod's high-level controller continuously tracks the center of mass and body pose choosing only to take a step when the center of mass is perturbed sufficiently from the center of the stance feet.

To predict the body orientation of the hexapod, a simple but effective complimentary filter is implemented. The complimentary filter uses six (or the number of limbs) inertial measurement units (IMUs) which are housed in the SEA modules that are rigidly fixed to the body (where the limbs meet the body). The redundant sensory information is advantageous in reducing noise in the IMU feedback by averaging over the six IMUs ( $\ddot{\mathbf{x}}_{\text {avg }}$ and $\dot{\boldsymbol{\theta}}_{\text {avg }}$ ). The averaged accelerometer data is then fed to the complimentary filter equation (1) which is a weighted sum of the accelerometer and gyro data.

$$
\begin{gathered}
\boldsymbol{\theta}_{\text {pose }}=W_{\text {gyro }}\left(\boldsymbol{\theta}_{\text {pose }}+\dot{\boldsymbol{\theta}}_{\text {gyro }} * \delta t\right)+\left(1-W_{\text {gyro }}\right) \dot{\boldsymbol{\theta}}_{\text {accel }} \\
\dot{\boldsymbol{\theta}}_{\text {accel }}=\tan ^{-1}\left(\frac{z_{\text {accel }}}{x_{\text {accel }}}\right) * \delta t
\end{gathered}
$$

The complimentary filter runs on $\mathbf{S O}(3)$ converting the averaged IMU data to a $3 \times 3$ rotation matrix $R_{\text {pose }}$ which provides the body pose estimate.

In addition to estimating the pose of the body, the balancing controller requires knowledge of the hexapod's center of mass $(\mathrm{CoM})$ relative to the stance feet in the body frame and in the world frame. The center of mass for each of the arms and legs is first computed and then combined to calculate to total center of mass as done in eq. (3).

$$
\left[\mathbf{x}_{\text {CoM }}\right]_{\text {world }}=\mathbf{R}_{\text {pose }}^{-1} \sum_{i}^{s} \frac{m_{i} \mathbf{x}_{i}}{m}
$$

Here $s$ is the total number of limb segments with mass $m_{i}$ and $\mathbf{x}_{i}$ is the 3-D position of that limb segment's center of mass. $\mathbf{x}_{i}$ is found by plugging the joint encoder feedback angles into the forward kinematics map where each segment's center of mass frame can be extracted from the map. $m$ is the total robot mass.

\section{Balancing Controller}

Using a quasi-static approach, the balancing controller ensures that the vertical projection of the hexapod's center of mass, or the zero moment point (ZMP), is kept within the support polygon. The support polygon is calculated by the convex hull of the foot-ground contact points. The algorithm then checks that this ZMP is within a specified threshold of the support polygon's boundaries, taking a step in the appropriate direction if that condition is not satisfied. Stance legs are commanded joint torques that support the weight of the body. This is accomplished by applying a force-torque wrench at each stance foot with a force in the negative $\mathrm{z}$ direction equal to the weight of the robot $(\mathrm{mg})$ divided by the number of legs in stance phase $\left(n_{s l}\right)$. This force is then rotated into the body frame to account for uneven weight distribution during non-symmetrical body poses as shown by eq. (5). The Jacobian transpose method is then applied to solve for the joint torques of the stance limbs. 


$$
\begin{aligned}
& \boldsymbol{\tau}=\mathbf{J}^{T} \mathbf{f} \\
& \mathbf{f}=\left[\begin{array}{lllll}
\mathbf{R}_{\text {pose }}^{-1}\left[\begin{array}{lll}
0 & 0 & -\frac{m g}{n_{s l}}
\end{array}\right]^{T} \quad 0 \quad 00 & 0
\end{array}\right]^{T}
\end{aligned}
$$

When the ZMP moves close to an edge of the support polygon a step is taken in the direction of the external force using either an alternating tripod or wave gait. The flight limbs follow a minimum jerk spline trajectory with four way points that begin at the current foot position and end at the prescribed 'home' position. The middle two way points are used to shape the flight trajectory and step height.

Additionally, gravity compensation is used during a limb's flight phase for smooth trajectory following while maintaining low position gains. Gravity compensation uses the Jacobian transpose method to solve for joint torque in flight legs which cancel out the weight of the limb simulating zerogravity.

When traveling over sloped or rough terrain pose correction is necessary to maintain body orthogonality relative to gravity. This is achieved by using the FSR foot sensors to determine when a stepping foot contacts the ground then observing the position of each feet relative to the body frame and compensating for the body's gravitational orientation. (6).

$$
\left(\mathbf{x}_{\text {foot }}^{B}\right)_{i}^{\prime}=\mathbf{R}_{\text {pose }}\left(\mathbf{x}_{\text {foot }}^{B}\right)_{i}
$$

To improve stance compliance and sensitivity to external forces acting on the body, a stance compliance term $\rho \in$ $[0,1]$ is introduced. The controller uses joint angle feedback to modify its commanded foot position using a weighted average of the commanded foot position and the current feedback position as shown by eq. (7).

$$
\left(\mathbf{x}_{\text {foot }}\right)_{i, c m d}=\rho\left(\mathbf{x}_{\text {foot }}\right)_{i, c m d}+(1-\rho)\left(\mathbf{x}_{\text {foot }}\right)_{i, f b k}
$$

The balancing controller continuously runs during operation and responds to take steps when the robot's CoM is displaced. The balancing controller works the same regardless of if the CoM was displaced from an external force or if it was displaced from a direct command using the joystick. In this way the robot is autonomous and tele-operable at the same time.

\section{QUADRUPED: ROLLER WALKER}

The quadruped, roller-walker, achieves its multi-modal mobility by being able to walk or roll on passive wheels. Multi-modal mobility offers advantages in terrain navigation by adjusting or adapting gait to best suit a given terrain. On rough terrains Snake Monster Roller-Walker can use a wave gait to prioritize stability and navigability. On flat ground or paved surfaces the quadruped can switch to roll mode which prioritizes energy efficiency and reduces cost of transport.

Similar to the hexapod, a redundant IMU complimentary filter is used as a state estimator to determine body orientation. The controller then applies force-torque wrenches at the feet to compensate for the weight of the body using the jacobian transpose method in eq. (4).

In walking mode where the rubber feet make up ground contact, the quadruped uses a balancing controller similar to that of the hexapod. The high-level controller tracks the center of mass and takes steps accordingly to maintain a ZMP within the support polygon.

In rolling mode the passive wheel becomes the ground contact element and the ankle joint uses body pose and leg kinematics to maintain parallelism between the wheel axis and the ground throughout the gait. The quadruped undulates its limbs, tracing a symmetric leg trajectory defined by $d(t)$ and $\theta(t)$, the time dependent polar coordinates of the foot [17].

$$
\begin{gathered}
d(t)=d_{o f f s e t}+d_{0}(\sin (\omega t+3 \pi / 2)+1) \\
\theta(t)=-\theta_{0} \sin (\omega t+3 \pi / 2+\phi)
\end{gathered}
$$

The foot position is a function of gait cycle percentage (or time) where $t=[0,2]$ for a period of 2 seconds. Here four control parameters are used to modulate the leg trajectory. $d_{0}$ and $\theta_{0}$ are amplitudes of sinusoidal oscillation in the normal and tangential directions of the passive wheel, respectively. $\omega$ is the angular velocity of oscillation, and $\phi$ is the phase difference between normal and tangential oscillations. A phase difference of $\phi_{f r}=3 \pi / 2$ radians between the front and rear legs is introduced to minimize velocity fluctuation at steady-state [17]. Turning is accomplished by increasing or decreasing $d_{0}$ to one pair/side of legs relative to the other side. For example to turn right the foot trajectory of the legs on the right side of the body will use a $d_{0}$ less than that of the left side.

\section{BIPED}

\section{A. State Estimation}

To achieve robust active balancing and walking an accurate knowledge of the biped's state variables must be estimated. Snake Monster biped uses the same complimentary filter as the hexapod to determine gravitational orientation of the body. In the case of the biped only 4 redundant IMU's are used. The 4 IMUs are located in the most proximal joint of each limb which is rigidly connected to the body.

\section{B. Balancing Controller}

1) Ankle Torque Strategy: The balancing controller is developed by sequentially combining 3 control strategies for incremental improvement in Snake Monster's balancing abilities. First, a simple ankle torque strategy is implemented by commanding joint torques to the ankle that oppose the moment induced by gravity acting at the robot's center of mass [25]. To calculate the desired ankle torques, the robot's total center of mass with respect to the stance feet in the world frame must be known. The ankle torques are calculated by

$$
\boldsymbol{\tau}_{\text {ankle }}=\mathbf{R}_{\text {pose }}^{-1}\left(\mathbf{x}_{C o M}-\mathbf{x}_{\text {foot }}\right) \frac{m g}{n_{s l}}
$$


2) Arm Controller: The ankle torque strategy is then augmented by an arm controller which uses the position of the arm's center of mass to shift the center of mass of the whole robot. This allows the balancing controller to bring the vertical projection of robot's total center of mass back within the support polygon in cases where it may temporarily have been displaced. The addition of arms allows for the effects of both dynamic inertial forces and the static shifting of the center of mass to aid the ankle torque strategy in stabilizing and balancing the biped.

Rather than the computationally expensive zero-momentpoint, center of mass trajectory optimization, the arm controller uses a simple PID controller. The PID controller moves its arms incrementally in the lateral and longitudinal planes based on the angle $(\phi)$ between the gravity vector and the vector connecting the center of pressure with the biped's center of mass.

$$
\phi=\mathbf{R}_{\text {pose }}^{-1}\left(\mathbf{x}_{C o M}-\mathbf{x}_{\text {foot }}\right)
$$

Additionally, the inertial force effects by dynamically moving the arms can be calculated and used to stabilize the body although this method was not implemented during the initial experiments done in this study. A simplified model of the inertial forces, assuming the arms are a point mass, can be computed as done in eq. (12).

$$
\boldsymbol{\tau}_{\text {arm }}=-m_{\text {arm }} \ddot{\mathbf{x}}_{\text {arm }}\left(\mathbf{x}_{\text {arm }}-\mathbf{x}_{C o M}\right)
$$

3) Virtual Model Force Control: The controller is then further augmented by the addition of a combined position and virtual model force controller [24]. The controller commands joint positions for the biped to stand in a basic 'home' pose. Additionally, the control strategy commands joint torques in the legs to achieve a desired virtual spring-damper between the ground and the body. The joint torques are determined using the Jacobian transpose method.

Similarly, gravity compensation is implemented during flight phase of a step to achieve weightlessness in the leg for better trajectory following. Gravity compensation is computed using a $6 n \times m$ Jacobian and a $6 n \times 1$ forcetorque wrench, where $n$ is the number of limb segments and $m$ is the number of joints (DoF) in the limb. Using the modified Jacobian transpose and force-torque wrench the joint torques to generate weightless limbs in flight can be calculated by equation (4).

\section{MANIPULATOR ARM}

In addition to the three different legged configurations (hexapod, quadruped, and biped) a 6-DoF arm, shown in Fig. 7 , with a gripper end-effector was configured to demonstrate the manipulation capabilities of these series elastic actuator modules. The force sensing gripper module at the distal end of the arm demonstrates yet another type of specialized module that interfaces seamlessly with the series elastic actuator modules. Using combined position, velocity, and force control very repeatable and dynamic motions are shown to be possible.

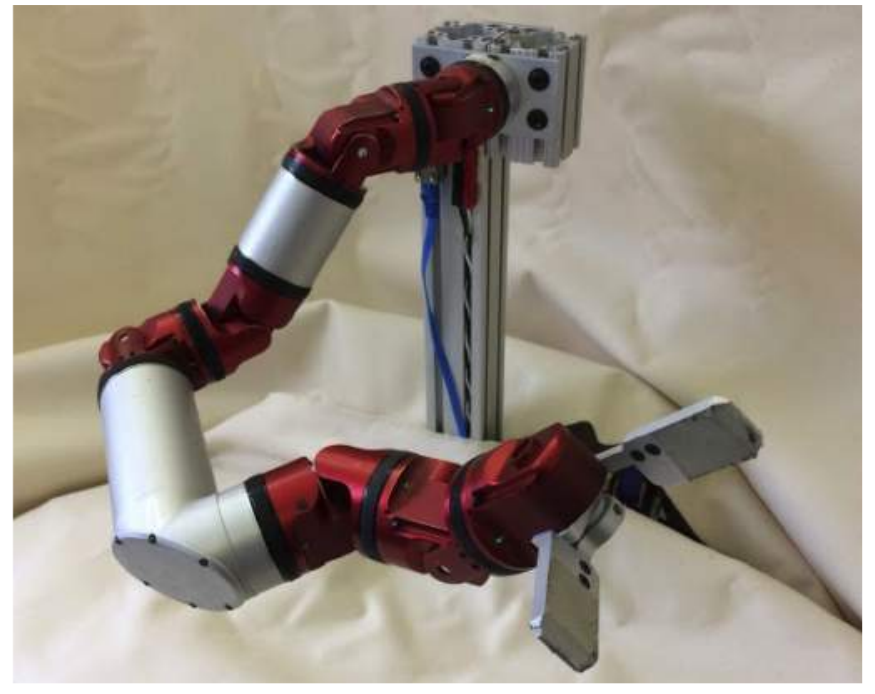

Fig. 7. Demonstration of a 6-DoF arm with force sensing gripper.

\section{Preliminary Results}

\section{A. Hexapod}

Snake monster has been deployed on indoor and outdoor terrains of varying roughness including pavement, grass, snow covered rocky grounds, and up stairs [27][28]. Currently, it is most capable on flat surfaces and has very dexterous pose and orientation control. Snake Monster walks with a stable alternating tripod gait in all directions. Experiments were conducted to evaluate the performance variations between the two control strategies: position vs position-force.

Cost of transport was estimated by measuring the electrical energy consumed for walking a distance of 7 meters over flat ground with walking parameters (forward velocity, step height, stance width, stance height, step period) all held constant. An average of 10 tests was taken per experiment; the results are summarized in Table I.

Feedback was collected at $200 \mathrm{~Hz}$ from each joint actuator which provided operating voltage and current draw per time step. Power was calculated using $P=I V$ and averaged over the 10 trials in the test.

In terms of disturbance handling and sensitivity to the environment the two control modes demonstrated noticeably different behavior. Sensitivity was tested by varying values of $\rho$ while applying measured forces to the body to determine the minimum amount of force required for the balancing controller to initiate a step. Results are shown in Table I.

The position control method was limited in responsiveness to external disturbances and did not easily take steps when being pushed or pulled. This intuitively makes sense as the position gains were increased and thus the entire robot would move when being pushed or pulled as opposed to just the body moving and the legs maintaining static and constant footholds in the world frame. For combined position-force control, as $\rho$ in eq. (7) is decreased from 1 the stance becomes incrementally more compliant and responsive to disturbances. However, if $\rho$ is decreased beyond about 0.6 , 
TABLE I

\begin{tabular}{lcccc}
\hline $\begin{array}{l}\text { Control } \\
\text { Strategy }\end{array}$ & $\begin{array}{c}\text { Stance } \\
\text { Comp. }(\rho)\end{array}$ & $\begin{array}{c}\text { Disturbance } \\
\text { Sensitivity } \\
{[\mathrm{N}]}\end{array}$ & $\begin{array}{c}\text { Cost of } \\
\text { Transport } \\
{[\mathrm{kJ} / \mathrm{m}]}\end{array}$ & $\begin{array}{c}\text { Max } \\
\text { Obstacle } \\
\text { Height }[\mathrm{m}]\end{array}$ \\
\hline Position & 0.6 & 5.3 & & \\
& 0.85 & 11.1 & $0.314 \pm .022$ & 0.065 \\
\hline Position- & 1.0 & $>>$ & & \\
Force & 0.6 & 3.3 & & 0.15 \\
\hline
\end{tabular}

walking performance degrades significantly as the limb no longer follows its defined trajectories with adequate accuracy and the gait looks visibly sloppy. Experimentation with varying values of $\rho$ found that $\rho=0.85$ seemed to produce the best balance of disturbance sensitivity and walking performance. Future studies will aim to optimize $\rho$ for a prescribed cost function dependent on cost of transport and disturbance sensitivity.

Additionally, force control also demonstrates significant mobility advantages over position control by allowing limbs to more easily conform to unmodeled changes in the terrain. Preliminary tests have shown that tele-operation using the position-force control can navigate over an obstacle with max height of 6 inches as opposed to position only control which can surmount 3-4 inch obstacles.

\section{B. Quadruped}

Initial roller-skating locomotion demonstrated maximum speeds of up to $0.5 \mathrm{~m} / \mathrm{s}$. In rolling mode the minimum turning radius was measured to be 0.6 meters. The cost of transport in rolling mode is approximately $0.047 \mathrm{~kJ} / \mathrm{m}$ while walking mode is significantly higher at $0.22 \mathrm{~kJ} / \mathrm{m}$. With optimization of the gait parameters, higher speed, increased stability, and a reduced turn radius is certainly feasible. A video of the roller-walker can be seen at the following link: https://www.youtube.com/watch?v=Vhgft53qfDQ

\section{Biped}

After implementing each incremental strategy of the balancing controller a series of experiments were conducted to test the ability of the biped to recover from externally applied disturbances. The experiments focused primarily on longitudinal stability and ignored active lateral stabilization because the support polygon is inherently larger in the lateral direction than the longitudinal direction.

The ankle torque strategy alone was capable of stabilizing itself under an applied 1 to $1.25 \mathrm{~N}-\mathrm{m}$ of torque about the foot (approximately a $2 \mathrm{~N}$ force at the upper body). A larger disturbance torque was able to be applied if the impulse of the applied force was reduced (e.g. the duration of the force increased). When the ankle torque gains are increased the biped can react faster and thus stabilize under disturbances on the order of 1.5 times larger. However, the controller will often overshoot creating too much momentum in the initial correcting direction causing the biped to fall over in the opposite direction of the initial disturbance force. Sometimes

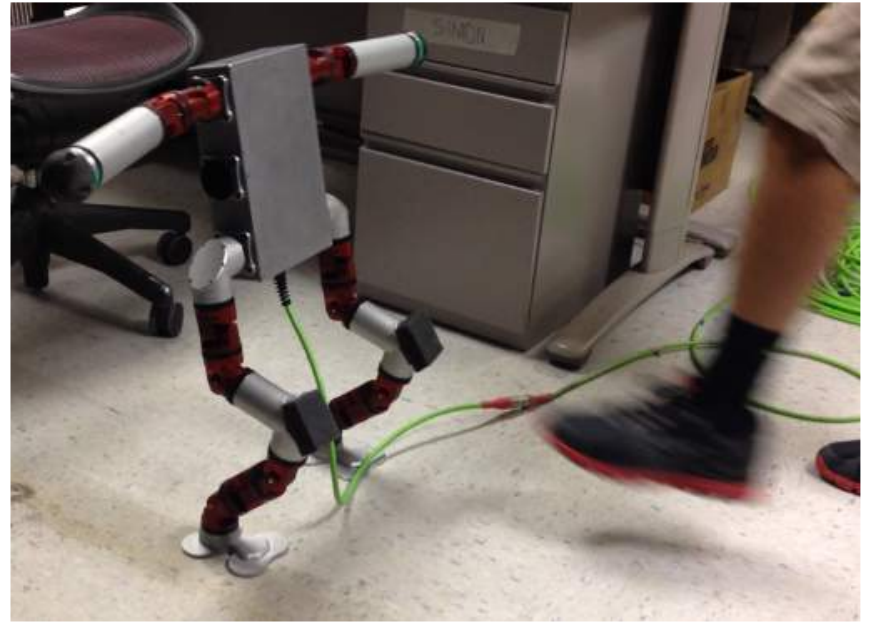

Fig. 8. Snake Monster Biped using ankle torque strategy and arm control to recover its balance from a kick.

in the intermediate range between a $2-3 \mathrm{~N}$ disturbance the biped will oscillate a few times around the equilibrium (balanced) position before settling.

After adding the arm control to the ankle torque strategy significantly larger disturbances (order of 1.5 to 2) times the can be reacted to. Further optimization of gains and the implementation of the dynamic inertial effect arm controller should drastically improve the robustness and consistency of balancing performance over even larger disturbance forces.

A dimensionless balancing performance ratio $\nu$ relates robot's weight to the max disturbance force, $F_{\max }$, it is capable of recovering from. The ratio is introduced to compare the performance of other biped/humanoid robots. Snake Monster Biped was calculated to have a performance ratio of 0.044 . Similar 1-legged standing balance (0-step) push recovery experiments have been conducted on other biped robots such as Atlas estimated to have $\nu=.055$ [26] and M2V2 with $\nu=0.048$ [32]. For reference an average human weighing about $80 \mathrm{~kg}$ has $\nu=0.051$ [31].

Additional experiments attempted to stabilize the biped in 1-legged balancing. These experiments were not successful which can be mainly attributed to the configuration of the biped legs which are separated by the width of the entire body making it difficult to position the biped's center of mass directly over one leg. The bipeds left to right hip distance needs to be decreased to improve both 1-legged balancing and walking where the center of mass needs to switch between legs quickly without large leg motions. A video of balancing experiments can be seen at: https://www.youtube.com/watch?v=7CciHJ6SP_o

\section{Manipulator Arm}

Three simple, yet highly demonstrative, experiments are conducted using a manipulator arm of various configurations and degrees of freedom. Videos of each experiment can be seen at their corresponding links.

In experiment one, the base joint of an arm is being commanded to move at full speed, and switch 
directions if a torque limit is exceeded. The sensitive torque sensing allows the joint to switch directions even when it encounters very light interference. https://www.youtube.com/watch?v=yUZVSQ $0 \mathrm{HtI}$

In experiment two, the repeatable motion is enabled by compensating for the effects of gravity and acceleration on each link of the arm. The series elastic actuation and torque control at each joint enable these high-speed maneuvers with low-bandwidth control. In this video, the command frequency was deliberately slowed to $25 \mathrm{~Hz}$, down from the maximum command frequency of $1 \mathrm{kHz}$. https://www.youtube.com/watch?v=Jb1-Mdklw1I

Experiment three is a demonstration using a 5 degreeof-freedom arm to eat lunch. The waypoints for the arm's motion were recorded by manually moving the robot through key poses and then played back and forth to get the repeated motions shown in the video. The entire demo, from teaching the waypoints through the execution of both sets of motions, took about 15 minutes to program. https://www.youtube.com/watch?v=wTCXDkW3ziQ

\section{CONClusion And Future Work}

Our modular architecture allows for simple and easy integration of modules of any type including but not limited to actuation, sensing, or passive/structural segments. These robotic actuators provide the fundamental building blocks for rapid development of future robots for mobility and manipulation. Any module design consisting of sensors, actuators, and mechanisms is compatible with the other modules so long as the design adheres to the same electromechanical interface and software protocol.

Additional modules being developed include a head module with IP cameras and a 2-D laser distance sensor which will be used for implementation of computer vision to map local terrain and optimize locomotion using footstep planning. Other multi-modal mobility leg end-effector modules such as driven wheels and micro-spine grippers are also being designed to further enhance the mobility of Snake Monster on varying or extreme terrains.

With the ability to reconfigure, modify, and build generalized or highly specialized modules, we have developed a new class of modular robots.

\section{ACKNOWLEDGMENT}

The authors would like to acknowledge the members of the Biorobotics lab for their contributions to this work. This work was funded by the DARPA M3 Program and an NDSEG Fellowship.

\section{REFERENCES}

[1] M. Piccoli, S. Revzen, M. Yim, "SEAL Pack Versatile, Portable, and Rapidly Deployable SEa, Air, and Land vehicle.

[2] M. Sugisaka, "Working robots for nuclear power plant disasters," in Digital Ecosystems and Technologies Conference (DEST), 2011.

[3] T. Fukuda and S. Nakagawa, "Dynamically reconfigurable robotic system," in Robotics and Automation, 1988.

[4] A. Wolf, H. B. Brown, R. Casciola, A. Costa, M. Schwerin, E. Shamas, and H. Choset, "A mobile hyper redundant mechanism for search and rescue tasks," in Intelligent Robots and Systems, 2003.(IROS 2003).
[5] M. Travers, H. Astley, P. Schiebel, C. Gong, D. Goldman, and H. Choset, "Constrained Whole-Body Compliance."

[6] J. Mankins, "Technology Readiness Levels - A White Paper." Advanced Concepts Office, Office of Space Access and Technology, NASA, 1995

[7] M. Yim, K Roufas, D. Duff, Y. Zhang, C. Eldershaw, S. Homans, "Modular Reconfigurable Robots in Space Applications."

[8] S. Yi, S. McGill, L. Vadakedathu, Q. He, I. Ha, M. Rouleau, D. Hong, and D.D. Lee, "Modular Low-Cost Humanoid Platform for Disaster Response," 2014 IEEE/RSJ IROS. Chicago, IL, USA.

[9] IEEE. (2015, June. 6). "A Compilation of Robots Falling Down at the DARPA Robotics Challenge." [YouTube video]. Available: https://www.youtube.com/watch? $\mathrm{v}=\mathrm{g} 0 \mathrm{TaYhjpOfo}$.

[10] IEEE. (2015, June. 5). "CHIMP Robot Falls Down, Gets Up at DARPA Robotics Challenge." [YouTube video]. Available: https://www.youtube.com/watch?v=du $4 \mathrm{tUPu} 2 \mathrm{eU}$.

[11] IEEE. (2015, April. 17). "Snake Monster Hexapod Robot." [YouTube video]. Available: https://www.youtube.com/watch?v=rM6' 1TeS6no.

[12] M. Raibert. "Legged Robots that Balance." MIT, 1986.

[13] D. Koditschek. Laboratory on Legs: An Architecture for Adjustable Morphology with Legged Robots.

[14] S. Kim. Bio-inspired Robot Design with Compliant Underactuated Systems. Doctoral Thesis, 2008.

[15] M. Murphy, A. Saunders, C. Moreira, A. Rizzi, and M. Raibert, 'The LittleDog Robot,"International Journal of Robotics Research, 2010.

[16] J. Rebula, P. Neuhaus, B. Bonnlander, M. Johnson, J. Pratt, "A Controller for the LittleDog Quadruped Walking on Rough Terrain," IEEE International Conference on Robotics and Automation, 2007.

[17] G. Endo, S. Hirose. Study on Roller-Walker: Energy Efficiency of Roller-Walk. 2011 IEEE ICRA. Shanghai, China.

[18] S. Ford, D. Rollinson, A. Willig, and H. Choset, "Online Calibration of a Compact Series Elastic Actuator." 2014 American Control Conference, Portland, USA.

[19] D. Rollinson, H. Choset. Design and Architecture of a Series-Elastic Snake Robot.

[20] D. Rollinson. Control and Design of Snake Robots. Thesis, 2014.

[21] J. Urata. Thermal Control of Electrical Motors for High-Power Humanoid Robots. 2008.

[22] D. Rollinson, H. Choset. Torque Control Strategies for Snake Robots.

[23] J. Pratt. Series Elastic Actuators for Legged Robots.

[24] J. Pratt, C. Chew, A. Torres, P. Dilworth, G. Pratt, "Virtual Model Control: An Intuitive Approach for Bipedal Locomotion" The International Journal of Robotics Research Vol. 20 No.2, 2001.

[25] D. Nenchev and A. Nishio, "Experimental Validation of Ankle and Hip Strategies for Balance Recovery with a Biped Subjected to an Impact," 2007 IEEE International Conference on Intelligent Robots and Systems. San Diego, 2007.

[26] Atlas Update. [Online]. Available: https://www.youtube.com/watch?v=SD6Okylclb8

[27] Snake Monster Hexapod Robot. [Online]. Available: https://www.youtube.com/watch?v=rM6 ITeS6no

[28] Snake Monster Stair Ascent. [Online]. Available: https://www.youtube.com/watch?v=UrjP3WB3VsY

[29] J. Urata, K. Nshiwaki, Y. Nakanishi, K. Okada, S. Kagami, and M. Inaba. "Online Decision of Foot Placement Using Singular LQ Preview Regulation," IEEE-RAS International Conference on Humanoid Robots. Bled, Slovenia, 2011.

[30] B. Stephens, C. Atkeson. "Push Recovery by Stepping for Humanoid Robots with Force Controlled Joints." IEEE-RAS International Conference on Humanoid Robots, Nashiville, TN, USA, 2010.

[31] Mille, M-L., Rogers, M.W., Martinez, K., Hedman, L.D., Johnson, M.E., Lord, S.R., and Fitzpatrick, R.C., "Thresholds for inducing protective stepping responses to external perturbations of human standing", Journal of neurophysiology, vol 90, no. 2, pp 666-674 2003

[32] J. Pratt., T. Koolen, et al. "Capturability-based analysis and control of legged locomotion, Part 2: Application to M2V2, a lower-body humanoid" International Journal of Robotics Research, 2012. 